\title{
Effects of Parent Training Programmes on Parents' Sense of Competence in a General Population Sample
}

\author{
Hans O. Löfgren ${ }^{1}$, Solveig Petersen ${ }^{1,2}$, Karin Nilsson ${ }^{1}$, Mehdi Ghazinour ${ }^{3} \&$ Bruno Hägglöf ${ }^{1}$ \\ ${ }^{1}$ Child and Adolescent Psychiatry, Department of Clinical Sciences, Umeå University, Sweden \\ ${ }^{2}$ Epidemiology and Global Health, Department of Public Health, Umeå University, Sweden \\ ${ }^{3}$ Police Academy, Umeå University, Sweden \\ Correspondence: Hans O. Löfgren, Child and Adolescent Psychiatry, Department of Clinical Sciences, Umeå \\ University, S-90187, Umeå, Sweden. Tel: 46-730-92-9795. E-mail: hans.lofgren@umu.se
}

Received: December 9, 2016 Accepted: December 29, 2016 Online Published: January 11, 2017

doi:10.5539/gjhs.v9n7p24 URL: http://dx.doi.org/10.5539/gjhs.v9n7p24

\begin{abstract}
Introduction: This longitudinal case-controlled study examined the effects of universal parent-focused interventions on parents' perceived competence in terms of parental efficacy and satisfaction.

Method: The study sample consisted of parents from northern Sweden in the general population who participated in parent training programmes from 2010 to 2013, and a matched-comparison group. All parents had children aged $0-17$. Sense of competence was measured by the Parenting Sense of Competence Scale at pre- and post-intervention and six months after the intervention.

Results: The intervention group showed a statistically significant improvement in parental competence compared to the comparison group over time. The intervention itself had a significant effect on parental satisfaction, but the efficacy effect was not sustained when taking into account potential confounders.

Conclusions: Earlier studies indicate that parent training programmes enhance perceived parental competence amongst referred parents. The present study shows that parent training programmes applied in the general population may also enhance perceived parental satisfaction, suggesting that parent training programmes can be an important preventive strategy to enhance parental feelings of satisfaction in the wider population. The results suggest that parents who participate in parent training programmes might have a need to increase parental competence, based on lower scores than the comparison group, both before and after the intervention.
\end{abstract}

Keywords: Parent training programme, parenting sense of competence, parental support, parenting, universal intervention.

\section{Introduction}

Health and human capacity to access good or poor health are influenced by gender norms, roles and relations, and other conditions in which people are born, grow, work, live and age. The wider the disparity is between different social groups, the greater the inequity in their ability to obtain good health and health care (WHO, 2016). The majority of Western children rate their health as good ( $83 \%$ average, both genders). However, older adolescents tended to report poor or fair health more often, with the effect being stronger amongst girls across all age groups. Gender differences were significant at age 11 in only a few countries and regions, but were significant for nearly all at age 15. Differences increased with increasing age. Thirty-two out of forty-two countries and regions saw an increase of 10 or more percentage points of fair or poor health in girls between age 11 and 15, except for Finland and Wales, where the increase was also seen in boys (Inchley \& Currie, 2013). However, the levels of low general well-being have increased with age in recent decades. A number of studies and reports indicate that symptoms such as depression, anxiety and insomnia are becoming increasingly common amongst young people, especially girls (Knopf, Park, \& Mulye, 2008; Merikangas et al., 2010; National Board of Health and Wellfare, 2009; Schwarz, 2009). The proportion of girls with such problems had an increased risk (odds ratio of 1.42 and $95 \%$; confidence interval between 1.20 and 1.67) of subjective health complaints in one-adult households compared with girls in two-adult households. Boys followed girls' upward trend, but far fewer reported feeling sadness and anxiety (Carlerby, Viitasara, Knutsson, \& Gillander Gadin, 2011; Swedish National Institute of Public Health, 2014). In 2011, in a large, randomised community setting $(\mathrm{n}=3$ 060), the incidence of non-suicidal self-injury (NSSI) was 
$35.6 \%$ of adolescents ( $56.2 \%$ female) with at least one episode of NSSI during the last year, of which $6.7 \%$ met suggested DSM-5 (APA, 2013) criteria for a potential NSSI disorder diagnosis (Zetterqvist, Lundh, Dahlström, \& Svedin, 2013). The majority of young people do not seek professional help for NSSI, which includes seeking medical help after an overdose. The majority of young people studied do, however, seek help from social networks, which most commonly comprise peers. Stressors in the family, including parent-child relationship and communication not directly related to the child's psychological problems can negatively affect help-seeking behaviour (Michelmore \& Hindley, 2012). Conflicts in the family and aggressive parental behaviour during upbringing are related to children externalising behaviours (Carrère \& Bowie, 2012); internalising problems (Beardslee et al., 1988; von Knorring, Cloninger, Bohman, \& Sigvardsson, 1983); higher mortality (Lund, Christensen, Nilsson, Kriegbaum, \& Rod, 2014); delayed brain maturation (Whittle et al., 2016), and delayed development (Neece, Green, \& Baker, 2012).

\subsection{Previous Studies}

The majority of studies on PTPs investigate the effects of selective or indicated prevention (Furlong et al., 2012). A few have investigated the use of PTPs as universal prevention in general populations in low-risk settings, which have included studying the marital quality of PTP participants (Bodenmann, Cina, Ledermann, \& Sanders, 2008); brief interventions of three sessions (Hiscock et al., 2008); workplace-oriented PTPs (Sanders, Stallman, \& McHale, 2011), or the demographics of participants (Alfredsson \& Broberg, 2016). Parental psychosocial health can have a significant effect on the parent-child relationship, with consequences for the later psychological health of the child and the family. Barlow, Smailagic, Huband, Roloff, and Bennett (2014) found that group-based PTPs led to statistically significant improvements in depression, anxiety, stress, anger, guilt, and negative or harsh parenting practice, whether assessed by both parents or assessed independently. There were also positive improvements in confidence, satisfaction with partner relationship, parental mental health and positive parenting skills. The meta-study also indicates that PTPs produced a statistically significant reduction in child-conduct problems, whether assessed by parents or by independent observers.

Parenting sense of competence (PSOC) was studied by Pisterman (1992) in families of pre-schoolers with attention deficit hyperactivity disorder, and showed significant correlation between improvements in PSOC and decreases in the parental stress index and parents' perceptions of improvement in their children's behaviour. The results indicate that PTPs have a positive impact on parenting stress and sense of competence and were independent of improvements in actual parent and child behaviour assessed in the clinic.

Sense of competence has been shown to be an important link between parents' personality and parenting. Agreeableness and extraversion were related to lower levels of over-reactivity in the child and higher levels of warmth in the parent. Sense of competence completely mediated relations between personality and over-activity in the child and partially mediated relations between personality and warmth of the parent (de Haan, Prinzie, \& Dekovic, 2009).

\subsection{Theoretical Framework}

Globally, parenting practices have three major goals: (a) ensuring children's health and safety, (b) preparing children for life as productive adults, and (c) transmitting cultural values (APA, 2000). A high-quality parent-child relationship is critical for healthy development. Bronfenbrenner's (1986) theory of the family ecological system is still a valid framework, where social settings interact with each other and the individual parent. Contributing theories to that framework are Bowlby's attachment theory (2005), where the parent (or carer) is the preferable secure base for the child in its exploration of the world; Crittenden's (2011) extended theory of the dynamic-maturational model provides a map of parents' functional and dysfunctional behaviours. Parent-development theory by Mowder (2005) describes the social role that parents develop and change over time and how the social role relates to parenting activities.

Parental-training programmes are based on four related components: (a) a conceptual view about how to change social, emotional, and behavioural problems; (b) a set of principles and techniques that follow on from that conceptual view in order to decrease risk factors and increase protective factors so the needs of every family member are met; (c) development of specific skills in parents through practice, role play, and other active methods of training; and (d) integration of assessment and evaluation in treatment and treatment decision-making (Kazdin, 2008).

Self-efficacy relates to people's beliefs in their ability to successfully manage events that affect their lives. This basic belief is the foundation of human motivation, performance accomplishment, and well-being. Those with high self-efficacy who believe they can perform well are more likely to view demanding tasks as something to be 
mastered rather than to be avoided. Parents with a strong sense of self-efficacy view problematic situations as challenges to be overcome, while parents with weaker self-efficacy avoid demanding tasks, believing them to be beyond their capabilities (Bandura, Adams, \& Beyer, 1977).

Parental sense of competence is closely related to the construct of self-efficacy. The two constructs are often used interchangeably, as is apparent from the definition of parental self-efficacy as parents' self-referent estimations of competence in the parental role (Coleman \& Karraker, 2003; de Haan et al., 2009). Parents are motivated to attempt behaviour that they consider beneficial. The parental sense of competence refers to the degree to which the parent feels competent and confident in handling child-related problems (Johnston \& Mash, 1989).

The Swedish Government has a strategy that emphasises the importance of targeting children and families through public health initiatives. This strategy aims to increase health-related living habits that could have an impact on health development in later life (Swedish National Institute of Public Health, 2014). This strategy emerged from the governmental report "New tools for parents" (Bremberg, 2004). This report advocated compiling and disseminating knowledge to people working with families and making PTPs available for all parents. These PTPs are often originally designed for clinical purposes in UK and US cultures, and include their views on parenting and family values; however, popular PTPs have been adapted to Swedish culture and to general family problems. Leader-led and structured workshops about everyday parental problems seem to have a positive impact on helping parents to care for children with clinical problems (Barlow, Smailagic, Ferriter, Bennett, \& Jones, 2010). Such a strategy might contribute to invigorating parents (or carers) in the parental role, to ameliorate parent-child relationships and reduce the rate of mental-health problems amongst children and adolescents (Kazdin, 1997).

\section{$1.3 \mathrm{Aim}$}

The objective of this study is to investigate how PTPs delivered in a general population setting affect parents' sense of parental competence. It is expected that parents who participate in PTPs will improve their sense of parental competence.

\section{Methods}

\subsection{Research Design}

The design was longitudinal and the study was part of a programme by the Swedish National Institute of Public Health named "New tools for parents - suggestions to new forms of parental support" (Bremberg, 2004). Data collection from the intervention group started in April 2010, peaked in February 2012 and ended in December 2013. Data collection from the comparison group took place between June and December 2013. The intervention group completed an online questionnaire on three separate occasions, before the start of the first PTP meeting (T1), immediately after the last meeting (T2) and six months after the last meeting (T3). The comparison group filled in an identical paper version of the questionnaire at home, at the same intervals as the intervention group.

\subsection{Participants}

\subsubsection{Intervention Group}

All parents of children aged 0-17 participating in the intervention were recruited through a broadly distributed advertisement. The intervention group comprised 562 parents, including 28 couples, who agreed to participate in the study. Participants were later excluded from the study group for one or more of the following reasons: participation in more than one PTP, less than $75 \%$ participation in the PTP, the child turning 18 during the study period, a large proportion of missing data, previous participation in a PTP, or literacy difficulties. The participants in the PTP intervention group finally comprised 260 parents completing both $\mathrm{T} 1$ and $\mathrm{T} 2$, of whom 115 parents also completed T3.

\subsubsection{Comparison Group}

The comparison group was randomly selected from a government database and chosen according to the same distribution of children's ages as in the intervention group. A total of 5000 invitations were sent and, 1209 (24.2\%) questionnaires were returned (T1). At T2, 103 participants were excluded from the comparison group for the same reasons as in the intervention group. This left 719 in the comparison group at T2, from which 115 matched-comparison participants were drawn for analysis, as described in the methods section. The identified socio-demographic difference between the intervention group and the comparison group at baseline was that the intervention group had younger parents (mothers $t=-3.21, p=.002$; fathers $t=-2.62, p=.01$ ) and more single parents $\left(c h i^{2}=4.28, p=.04\right)$. 
Table 1. Demographic variables at baseline

\begin{tabular}{|c|c|c|c|}
\hline Baseline variable & & $\begin{array}{l}\text { Intervention } \\
\text { group }(\mathrm{n}=115)\end{array}$ & $\begin{array}{l}\text { Comparison } \\
\text { group }(\mathrm{n}=115)\end{array}$ \\
\hline \multicolumn{4}{|l|}{ Child characteristics } \\
\hline Age of child M (SD) & From 0 to 17 years & $5.04(3.18)$ & $5.43(4.35)$ \\
\hline \multirow{2}{*}{ Child's gender N (\%) } & Boy & $61(56.0)$ & $61(54.5)$ \\
\hline & Girl & $48(44.0)$ & $51(45.5)$ \\
\hline \multirow{2}{*}{ Origin of child N (\%) } & In Sweden & $103(100)$ & $110(97.3)$ \\
\hline & Out of Sweden & $0(0)$ & $3(2.7)$ \\
\hline \multicolumn{4}{|l|}{ Parent/family characteristics } \\
\hline \multirow{2}{*}{ Gender of respondent $\mathrm{N}(\%)$} & Male & $13(11.3)$ & $14(12.3)$ \\
\hline & Female & $102(88.7)$ & $100(87.7)$ \\
\hline Age of fathers M (SD) & From 20 to 62 years & $36.81(5.98)^{*}$ & $38.79(6.35)^{*}$ \\
\hline Age of mothers M (SD) & From 20 to 60 years & $34.33(5.50)^{*}$ & $36.95(6.08)^{*}$ \\
\hline \multirow[t]{2}{*}{ Marital status N (\%) } & $\begin{array}{l}\text { Married/common-law } \\
\text { partner }\end{array}$ & $92(86.8)^{*}$ & $109(94.8)^{*}$ \\
\hline & Single & $14(13.2)^{*}$ & $6(5.2)^{*}$ \\
\hline Fathers' education N (\%) & Above high school & $53(56.4)$ & $63(60.6)$ \\
\hline Mothers' education N (\%) & Above high school & $73(71.6)$ & $94(82.5)$ \\
\hline \multirow{2}{*}{ Employment N (\%) } & Full-time & $83(75.5)$ & $80(70.2)$ \\
\hline & Part-time & $27(24.5)$ & $34(29.8)$ \\
\hline \multirow{2}{*}{ Origin of father N (\%) } & In Sweden & $101(93.5)$ & $102(92.7)$ \\
\hline & Out of Sweden & $7(6.5)$ & $8(7.3)$ \\
\hline \multirow{2}{*}{ Origin of mother N (\%) } & In Sweden & $100(92.6)$ & $104(90.4)$ \\
\hline & Out of Sweden & $8(7.4)$ & $11(9.6)$ \\
\hline
\end{tabular}

Note. ${ }^{*}$ difference $p<.05$.

\subsection{Interventions}

The PTPs included five programmes, Active Parenting, The Community Parent Education Programme (COPE), The Family Workshop, The Family Lab, and the "I love you, but you drive me nuts" programme. All targeted the general population and had similar delivery times (about 20 hours) and intervention themes. The PTPs were mostly delivered on a weekly basis at the leader's workplace during the evening. All leaders of the PTPs had completed the education requirements for each programme that were provided through the organisations responsible for the programmes.

Active Parenting was the first manual-based parenting programme using videos in Sweden. This programme is delivered in three versions: Active Toddler Parents ( $0-2$ years), Active Parenting Today ( $2-12$ years), and Active Parenting Today for Parents of Teens (13-17 years). Active Parenting was developed by Michael Popkin and is based on Adler's theories of development and learning (Popkin, 2014).

COPE is a manual-based education programme with videos and problem-solving discussions in "fish-bowls" (i.e. small groups). COPE was developed by Professor Cunningham in Canada and is available for three age groups: Toddlers ( $0-2$ years), 3-12 years, and teenagers (13-17 years). The programme is based on social-learning theory, social-cognitive psychology, and family-system theory (Dubow et al., 2010).

The Family Workshop was developed by the Centre of Prevention for Stockholm City and is a manual-based communication programme using videos, which targets parents of children aged between 3 and 12. It has been observed that the discussions are less guided than in other PTPs, although the programme is based on the aforementioned common theories and on clinical experience (Bremberg, 2004). 
The Family Lab was developed by the family therapist Jesper Juul and is conducted by lecture and discussion based on Kempler's Gestalt family theory (Kempler, 1974). The programme is dominated by dialogue enhancement between the responsible parent and the competent child (Schill, 2013).

"I love you but you drive me nuts" is a PTP for parents of teenagers. The programme is based on a Swedish manual that focuses on guided group discussions and practical homework tasks (Kimber \& Molgaard, 2009). This programme was based on the Iowa Strengthening Families Programme (Cui \& Donnellan, 2009).

\subsection{Measures}

Sense of competence was measured by the PSOC. The PSOC version used in the current study has 16 assertions that reflect parental self-perceptions of skills and knowledge regarding parental functions (Johnston \& Mash, 1989). These assertions yield a satisfaction subscale that captures how pleased parents are with their parenting role and an efficacy scale that captures how parents perceive their competence in the parenting role (Ohan, Leung, \& Johnston, 2000).

The nine items about frustration in the satisfaction subscale are negatively scored from " $1=$ strongly agree" to " $6=$ strongly disagree" and then summed. The seven items about positive parental esteem in the efficacy subscale are positively scored from " $1=$ strongly disagree" to " $6=$ strongly agree" and then summed. Higher scores indicate higher perceived parental efficacy and satisfaction. In this study, the Cronbach's $\alpha$ for the satisfaction subscale was .79 for fathers and .78 for mothers at T1. In the efficacy subscale, the Cronbach's $\alpha$ was .79 for fathers and .82 for mothers at T1. Comparison participants were matched to intervention participants by closest pre-scores on the sum of the two subscales.

Demographic data included the age and sex of the responding parent and child; parental marital status (dichotomised into married/partners and not married/partners), education level of the respondent and the spouse (dichotomised into less than and more than 12 years of education), origin of mother and father (born in Sweden and not born in Sweden), and child's place of birth (born in Sweden and not born in Sweden). There was also an additional question about whether or not an incident that might have a major impact on the family had occurred during the time of the PTP, or for the comparison group, between T1 and T2. Joint participation of both mother and father in the PTP was noted.

\subsection{Statistical Analyses}

Differences in socio-demographic characteristics between the intervention group and the comparison group were tested by chi $^{2}$ test or t-tests.

A paired t-test was conducted to estimate changes between PSOC subscale scores at T1 and scores at T2 and T3. An independent $t$-test was conducted on the difference between the intervention group and comparison group from $\mathrm{T} 1$ to T2 and from T1 to T3. The effect size was quantified by Cohen's $d$-value, where less than 0.2 is defined as a "small" effect, 0.5 as a "medium" effect, and 0.8 as a "large" effect (Cohen, 1988).

Univariate analysis of variance was performed to: adjust for the socio-demographics that differed between the intervention and comparison group; and to adjust for the difference in pre-score, considering ceiling effect and regression towards mean. Missing values for the PSOC scale were assessed by estimation maximisation techniques if there were fewer than three missing values in each subscale. The significance level was set to .05 .

All analyses were conducted using the IBM Statistical Package for the Social Sciences (SPSS) 21.0.0.1.

\subsection{Ethics}

The Regional Ethical Review Board in Umeå, Sweden approved the study (2010/249-31Ö). The study followed the APA Ethics Code.

\section{Results}

There was a statistically significant increase in the efficacy subscale in both the intervention group and the comparison group from T1 to T2 and from T1 to T3 (Table 2).

The intervention group's mean score on the satisfaction subscale was .92 points lower than the comparison group at T1. There was a statistically significant increase in the intervention group from T1 to T2 and from T1 to T3. There was also a statistically significant increase in the comparison group from $\mathrm{T} 1$ to $\mathrm{T} 3$, but not from $\mathrm{T} 1$ to $\mathrm{T} 2$. It is important to note that the intervention group's mean score on the efficacy subscale was 3.37 points lower than the comparison group at $\mathrm{T} 1$. 
Table 2. Paired t-tests of change on parental satisfaction and efficacy from T1 to T2 and T3, respectively

\begin{tabular}{|c|c|c|c|c|c|c|c|}
\hline & & \multirow{2}{*}{ Mean (SD) } & \multicolumn{4}{|c|}{ Differences within groups } & \multirow{2}{*}{ d-value } \\
\hline & & & Mean & SD & $\mathrm{T}$ & p-value & \\
\hline Intervention & T1 Efficacy & $26.35(5.19)$ & & & & & \\
\hline$(\mathrm{n}=107)$ & T2 Efficacy & $29.31(5.21)$ & 2.96 & 4.02 & 7.61 & $<.001$ & .74 \\
\hline$(\mathrm{n}=111)$ & T3 Efficacy & $29.02(5.45)$ & 2.84 & 4.43 & 6.75 & $<.001$ & .64 \\
\hline Comparison & T1 Efficacy & $29.72(4.34)$ & & & & & \\
\hline$(\mathrm{n}=109)$ & T2 Efficacy & $30.68(4.78)$ & .96 & 3.44 & 2.90 & .04 & .28 \\
\hline$(\mathrm{n}=107)$ & T3 Efficacy & $31.30(4.34)$ & 1.43 & 3.51 & 4.22 & $<.001$ & .41 \\
\hline Intervention & T1 Satisfaction & $38.21(6.30)$ & & & & & \\
\hline$(\mathrm{n}=105)$ & T2 Satisfaction & $41.52(5.55)$ & 3.32 & 5.12 & 6.64 & $<.001$ & .65 \\
\hline$(\mathrm{n}=113)$ & T3 Satisfaction & $41.93(6.02)$ & 4.07 & 6.43 & 6.74 & $<.001$ & .61 \\
\hline Comparison & T1 Satisfaction & $39.13(5.20)$ & & & & & \\
\hline$(\mathrm{n}=109)$ & T2 Satisfaction & $39.83(6.70)$ & .71 & 4.70 & 1.58 & .12 & .15 \\
\hline$(\mathrm{n}=112)$ & T3 Satisfaction & $40.13(6.00)$ & 1.14 & 4.64 & 2.59 & .01 & .25 \\
\hline
\end{tabular}

There was a statistically significant difference between the groups in favour of the intervention group in development from $\mathrm{T} 1$ to $\mathrm{T} 2$ and from $\mathrm{T} 1$ to $\mathrm{T} 3$, in both subscales (Table 3 ). The difference in raw effects sizes in favour of the intervention group in both subscales was medium size at $\mathrm{T} 2$, and small to medium size for efficacy in T3.

Table 3. Independent t-test of the difference in intervention and comparison group on parental satisfaction and efficacy between $\mathrm{T} 1$ to $\mathrm{T} 2$ and $\mathrm{T} 1$ to $\mathrm{T} 3$, respectively

\begin{tabular}{llllllll}
\hline & & \multicolumn{3}{c}{ Differences between groups } & & \\
\cline { 3 - 8 } & & Mean & Mean SD & t & df & p-value & d-value \\
\hline T1 to T2 & Diff Efficacy & 2.00 & 3.73 & 3.94 & 214 & $<.001$ & .54 \\
& Diff Satisfaction & 2.61 & 4.91 & 3.88 & 212 & $<.001$ & .53 \\
\hline T1 to T3 & Diff Efficacy & 1.41 & 3.97 & 2.61 & 208.16 & .01 & .35 \\
& Diff Satisfaction & 2.94 & 5.39 & 3.93 & 203.84 & $<.001$ & .53 \\
\hline
\end{tabular}

Note. A positive mean-value favouring the intervention group.

The pre-score was included in the ANOVA to control for regression towards mean, yet the pre-score effect was larger than expected $(t=-4.98)$. The analysis showed that parents who had participated in PTPs had a higher mean increase $(B=1.01)$ in efficacy at $\mathrm{T} 2$ than the comparison group when controlling for the demographic variables that differed according to Table 1 (parental age and marital status).

The small mean increase in efficacy at T3 $(B=0.06)$ was insignificant. Parents that participated in PTPs also had a higher mean increase in satisfaction $(B=2.41)$ and the effect remained after six months $(\mathrm{T} 3, B=2.31)$. No socio-demographic variable was significant in any of the four models of satisfaction, with T2 and T3 and efficacy $\mathrm{T} 2$ and $\mathrm{T} 3$. 
Table 4. ANOVA of difference in main effect between intervention group and comparison group, controlling for pre-score, parent age and marital status

\begin{tabular}{|c|c|c|c|c|c|c|}
\hline Model & $\operatorname{Adj} R^{2}$ & $F$ & Coefficients & $B$ & $t$ & $p$ \\
\hline \multirow[t]{5}{*}{ Efficacy T2 } & .15 & 8.23 & Intervention & .92 & 1.60 & .11 \\
\hline & & & Pre-score & -.26 & -4.75 & $<.001$ \\
\hline & & & Age fathers & -.02 & -.26 & .80 \\
\hline & & & Age mothers & -.01 & -.14 & .89 \\
\hline & & & Single parent & .92 & .92 & .36 \\
\hline \multirow[t]{5}{*}{ Satisfaction T2 } & .17 & 8.92 & Intervention & 2.25 & 3.22 & .001 \\
\hline & & & Pre-score & -.29 & -5.01 & $<.001$ \\
\hline & & & Age fathers & -.01 & .08 & .94 \\
\hline & & & Age mothers & -.06 & -.71 & .48 \\
\hline & & & Single parent & -1.06 & -.78 & .44 \\
\hline \multirow[t]{5}{*}{ Efficacy T3 } & .14 & 7.77 & Intervention & .09 & .15 & .88 \\
\hline & & & Pre-score & -.33 & -5.40 & $<.001$ \\
\hline & & & Age fathers & .01 & .06 & .95 \\
\hline & & & Age mothers & -.04 & -.63 & .53 \\
\hline & & & Single parent & -1.03 & -1.03 & .30 \\
\hline \multirow[t]{5}{*}{ Satisfaction T3 } & .25 & 14.79 & Intervention & 2.49 & 3.41 & .001 \\
\hline & & & Pre-score & -.43 & -7.16 & $<.001$ \\
\hline & & & Age fathers & .01 & .12 & .91 \\
\hline & & & Age mothers & -.03 & -.33 & .74 \\
\hline & & & Single parent & -.65 & -.49 & .62 \\
\hline
\end{tabular}

The standardised residuals from the regression analyses showed homoscedasticity for the satisfaction subscale, but not for efficacy. In the intervention group, there was no difference in either subscale for questionnaire distribution by paper, e-mail, or online; joint or non-joint participation by couples; participation frequency; or whether any major impact on the family was reported or not. The intervention group sample at T2 and T3 did not differ significantly from the dropout group in either of the subscales.

\section{Discussion}

This study examined the effects of PTPs on parents' sense of competence in a longitudinal experimental case-controlled design amongst parents in the general population. Some limitations might affect the results, such as design of the study. The municipality executive board did not allow a randomised controlled design, due to parents' freedom of choice; therefore, a case-controlled design was chosen. Another limitation is that the possibility of systematic bias from self-selection cannot be excluded, as reported in other studies (Lindsay, Strand, \& Davis, 2011).

The results show that parents completing the PTP experienced higher increases in parental satisfaction than the comparison group, both directly post-intervention and after six months. High parental satisfaction as measured by the PSOC scale has been shown to highly correlate with positive measures of child behaviour and parental well-being (Rogers \& Matthews, 2004).

Accordingly, a recent cross-sectional study of parental satisfaction (Salari, Wells, \& Sarkadi, 2014) showed that parental high-satisfaction scores related to low ratings of child behaviour problems. That study investigated Swedish parents in a general population of pre-school children with the same sample size as in the present study. Together, these results, and the finding that parents feel more satisfied with their role as a parent after participating in a PTP, indicate that PTPs may have additional positive implications for the well-being of parents and their children. However, this assumption requires further study.

The initial analysis indicated that the PTP also increased parental self-efficacy, (i.e. parents' beliefs in their ability 
to successfully manage parental events that affect their lives). However, this effect was not sustained when taking account of potential confounders. As a comparison from other studies, the pre-scores in efficacy were 27.63 (4.26) for a group of parents with adolescents who have autism spectrum disorders, and in satisfaction 39.19 (5.14), (Schultz, Stichter, Herzog, McGhee, \& Lierheimer, 2012). In a general population sample from pre-schools in Sweden, efficacy was 30.37 (4.77) and satisfaction 41.82 (6.49), (Salari et al., 2014). In the current study, the mean of both subscales was lower than for the two aforementioned studies (cf. Table 2).

In addition, the mean level of parental efficacy in this study's intervention group did not reach that of the comparison group, even after participation in the PTP. The impact of pre-scores in the ANOVA may indicate that parents with low initial scores had a better effect from the PTPs. In turn, this might indicate that parents in need of amelioration actively seek training groups if PTPs are offered. However, the increase in the mean level of satisfaction was higher than in the comparison group and remained so after six months.

A previous study of referred families has reported large effect sizes in self-rated parental efficacy and medium-effect sizes in parental satisfaction (Moretti \& Obsuth, 2009). These effect sizes were slightly higher than in the present study. The explanation for this might be an initially high level of problems being reported for the parents or children in that study. In the present study, an increase was found for the comparison parents in efficacy scores at T2 and T3, and in satisfaction at T3, which suggests improvement over time in parental competence, or simply that answering the questionnaire had a statistically significant learning effect.

An interesting finding was that it was only possible to capture less than a quarter of reasonable explanations in the variance of PSOC, as shown in the adjusted coefficient of determination, presented in Table 4. This might suggest that further investigation of the concepts of PTPs needs to include other components such as discussion climate, leader's charisma, or positive hints about practical child rearing. The current study adds to the view that the general population might be suitable for preventive action strategies. According to Rose (2008), a small change in the average in society will have a larger overall impact in reducing the total number of negative outcomes than by focusing just on the group that is most at risk for negative outcomes. Thus, the ability to enhance parental competence on a large scale with the majority of the general population might, in the long term, lead to fewer families with problems at the tail end of the normal distribution of parental competence. These findings indicate that PTPs are suitable for primary prevention interventions.

A strength of the current study was the use of a standardised measure of parental competence (PSOC) that has reported significant reliability and validity and assesses distinct aspects of parental self-esteem (Ohan et al., 2000). Another asset was the use of manualised intervention programmes that can be copied in other contexts. Furthermore, the intervention group leaders were well educated and skilled in their particular PTP.

\section{Conclusions}

Parental-training programmes as a universal preventive intervention may enhance the satisfaction of parents in the general population. However, the component efficacy in the PSOC instrument did not show an intervention effect in this study. The effects on children and on increased satisfaction that also positively affect problem-solving strategies, performance accomplishments and parental well-being need to be studied further; these conclusions need to be confirmed in studies with more restricted design.

\section{Acknowledgements}

Thanks are due to all parents and PTP leaders for their participation, Birgitta Törnqvist for statistical guidance, and research assistant Lena Grundberg for help with data collection.

\section{Competing Interests Statement}

The authors declare that they have no competing or potential conflicts of interest.

\section{Funding}

The study was funded by the Swedish National Institute of Public Health, grant numbers HF $2009 / 192$ (NKC2009/20) and HFÅ2010/95 (NKC2010/44), and the Fredrik \& Ingrid Thurings foundation.

\section{References}

Alfredsson, E. K., \& Broberg, A. G. (2016). Universal parent support groups for parents of adolescents: Which parents participate and why? Scandinavian journal of psychology, 57(2), 177-184. https://doi.org/10.1111/sjop.12278

APA. (2000). Encyclopedia of Psychology. In A. E. Kazdin (Ed.), Encyclopedia of Psychology (Vol. Institutions and Environments: Family, School, and Work, pp. 4128). Washington, DC: American Psychological 
Association.

APA. (2013). Diagnostic and Statistical Manual of Mental Disorders (DSM-5) 1000 Wilson Boulevard, Suite 1825, Arlington: American Psychiatric Association's

Bandura, A., Adams, N. E., \& Beyer, J. (1977). Cognitive processes mediating behavioral change. Journal of personality and social psychology, 35(3), 125. https://doi.org/10.1037/0022-3514.35.3.125

Barlow, J., Smailagic, N., Ferriter, M., Bennett, C., \& Jones, H. (2010). Group-based parent-training programmes for improving emotional and behavioural adjustment in children from birth to three years old. Cochrane Database Syst Rev, 3, CD003680. https://doi.org/10.1002/14651858.CD003680.pub2

Barlow, J., Smailagic, N., Huband, N., Roloff, V., \& Bennett, C. (2014). Group-based parent training programmes for improving parental psychosocial health. Cochrane Database Syst Rev, 5, CD002020. https://doi.org/10.1002/14651858.CD002020.pub4

Beardslee, W. R., Keller, M. B., Lavori, P. W., Klerman, G. K. Dorer, D. J., \& Samuelson, H. (1988). Psychiatric disorder in adolescent offspring of parents with affective disorder in a non-referred sample. Journal of Affective Disorders, 15(3), 313-322. https://doi.org/10.1016/0165-0327(88)90028-6

Bowlby, J. (2005). A secure base: Clinical applications of attachment theory (Vol. 393): Taylor \& Francis.

Bremberg, S. (Red). (2004). New tools for parents - suggestions to new forms of parental support. Sandviken.

Bronfenbrenner, U. (1986). Ecology of the family as a context for human development: Research perspectives. Developmental psychology, 22(6), 723. https://doi.org/10.1037/0012-1649.22.6.723

Carlerby, H., Viitasara, E., Knutsson, A., \& Gillander-Gadin, K. (2011). Subjective health complaints among boys and girls in the Swedish HBSC study: focussing on parental foreign background. Int J Public Health, 56(5), 457-464. https://doi.org/10.1007/s00038-011-0246-8

Carrère, S., \& Bowie, B. H. (2012). Like parent, like child: Parent and child emotion dysregulation. Archives of psychiatric nursing, 26(3), e23-e30. https://doi.org/10.1016/j.apnu.2011.12.008

Cohen, J. (1988). Statistical power analysis for the behavioral sciences. New York: Psychology Press.

Coleman, P. K., \& Karraker, K. H. (2003). Maternal self - efficacy beliefs, competence in parenting, and toddlers' behavior and developmental status. Infant Mental Health Journal, 24(2), 126-148.

Crittenden, P. M. (2011). Raising Parents (2nd ed.). Cullompton: Willan.

Cui, M., \& Donnellan, M. B. (2009). Trajectories of Conflict over Raising Adolescent Children and Marital Satisfaction. Journal of Marriage and the Family, 71(3), 478-494. doi: https://doi.org/10.1111/j.1741-3737.2009.00614.x

de Haan, A. D., Prinzie, P., \& Dekovic, M. (2009). Mothers' and fathers' personality and parenting: the mediating role of sense of competence. Developmental Psychology, 45(6), 1695-1707. https://doi.org/10.1037/a0016121

Dubow, E. F., Boxer, P., Huesmann, L. R., Shikaki, K., Landau, S., Gvirsman, S. D., \& Ginges, J. (2010). Exposure to conflict and violence across contexts: relations to adjustment among Palestinian children. Journal of Clinical Child and Adolescent Psychology, 39(1), 103-116. https://doi.org/10.1080/15374410903401153

Furlong, M., McGilloway, S., Bywater, T., Hutchings, J., Smith, S. M., \& Donnelly, M. (2012). Behavioural and cognitive-behavioural group-based parenting programmes for early-onset conduct problems in children aged 3 to 12 years. Cochrane Database Syst Rev, 2, CD008225. https://doi.org/10.1002/14651858.CD008225.pub2

Inchley, J., \& Currie, D. (2013). Growing up unequal: gender and socioeconomic differences in young people's health and well-being. Health Behaviour in School-aged Children (HBSC) study: international report from the, 2014(Part 2), Chapter 3.

Johnston, C., \& Mash, J. E. (1989). A Measure of Parenting Satisfaction and Efficacy. Journal of Clinical Child Psychology, 18(2), 8. https://doi.org/10.1207/s15374424jccp1802_8

Kazdin, A. E. (1997). Parent management training: Evidence, outcomes, and issues. Journal of the American Academy of Child \& Adolescent Psychiatry, $36(10), \quad$ 1349-1356. https://doi.org/10.1097/00004583-199710000-00016

Kazdin, A. E. (2008). Parent management training: Treatment for oppositional, aggressive, and antisocial 
behavior in children and adolescents: Oxford University Press.

Kempler, W. (1974). Principles of gestalt family therapy: A gestalt-experiential handbook: Kempler Inst.

Kimber, B. \& Molgaard, V. (2009). Älskade förbannade tonåring. Stockholm: Natur \& Kultur.

Knopf, D., Park, M. J., \& Mulye, T. P. (2008). The mental health of adolescents: A national profile, 2008. San Francisco, CA: National Adolescent Health Information Center.

Lindsay, G., Strand, S., \& Davis, H. (2011). A comparison of the effectiveness of three parenting programmes in improving parenting skills, parent mental-well being and children's behaviour when implemented on a large scale in community settings in 18 English local authorities: the parenting early intervention pathfinder (PEIP). BMC Public Health, 11, 962. https://doi.org/10.1186/1471-2458-11-962

Lund, R., Christensen, U., Nilsson-Juul, C., Kriegbaum, M., \& Hulvej-Rod, N. (2014). Stressful social relations and mortality: a prospective cohort study. Journal of Epidemiology and Community Health, jech-2013-203675.

Merikangas, K. R., He, J-P., Burstein, M., Swanson, S. A., Avenevoli, S., Cui, L., . . Swendsen, J. (2010). Lifetime prevalence of mental disorders in US adolescents: results from the National Comorbidity Survey Replication-Adolescent Supplement (NCS-A). Journal of the American Academy of Child \& Adolescent Psychiatry, 49(10), 980-989. https://doi.org/10.1016/j.jaac.2010.05.017

Michelmore, L., \& Hindley, P. (2012). Help - seeking for suicidal thoughts and self - harm in young people: A systematic review. Suicide and Life-Threatening Behavior, 42(5), 507-524. https://doi.org/10.1111/j.1943-278X.2012.00108.x

Moretti, M. M., \& Obsuth, I. (2009). Effectiveness of an attachment-focused manualized intervention for parents of teens at risk for aggressive behaviour: The Connect Program. Journal of Adolescence, 32(6), 1347-1357. https://doi.org/S0140-1971(09)00108-0 [pii] 10.1016/j.adolescence.2009.07.013

Mowder, B. A. (2005). Parent development theory: Understanding parents, parenting perceptions and parenting behaviors. Journal of Early Childhood and Infant Psychology, 1, 45.

National Board of Health and Wellfare. (2009). Public Health Report 2009. Västerås: Edita Retrieved from http://www.socialstyrelsen.se/Lists/Artikelkatalog/Attachments/8495/2009-126-71_200912671.pdf.

Neece, C. L., Green, S. A., \& Baker, B. L. (2012). Parenting stress and child behavior problems: A transactional relationship across time. American Journal on Intellectual and Developmental Disabilities, 117(1), 48-66. https://doi.org/10.1352/1944-7558-117.1.48

Ohan, J. L., Leung, D. W., \& Johnston, C. (2000). The parenting sense of competence scale: Evidence of a stable factor structure and validity. Canadian Journal of Behavioural Science-Revue Canadienne Des Sciences Du Comportement, 32(4), 251-261. https://doi.org/10.1037/h0087122

Pisterman, S., Firestone, P., Mcgrath, P., Goodman, J. T., Webster, I., Mallory, R., \& Goffin, B. (1992). The Effects of Parent Training on Parenting Stress and Sense of Competence. Canadian Journal of Behavioural Science-Revue Canadienne Des Sciences Du Comportement, 24(1), 41-58. https://doi.org/10.1037/h0078699

Popkin, M. H. (2014). Active Parenting: 30 Years of Video-Based Parent Education. The Journal of Individual Psychology, 70(2), 166-175. https://doi.org/10.1353/jip.2014.0014v

Rogers, H., \& Matthews, J. (2004). The parenting sense of competence scale: Investigation of the factor structure, reliability, and validity for an Australian sample. Australian Psychologist, 39(1), 88-96. https://doi.org/10.1080/00050060410001660380

Rose, G. (2008). Rose's strategy of preventive medicine: the complete original text (Vol. 1): Oxford University Press.

Salari, R., Wells, M. B., \& Sarkadi, A. (2014). Child behaviour problems, parenting behaviours and parental adjustment in mothers and fathers in Sweden. Scandinavian Journal of Public Health, 547-553. https://doi.org/10.1177/1403494814541595

Sanders, M. R., Stallman, H. M., \& McHale, M. (2011). Workplace Triple P: A controlled evaluation of a parenting intervention for working parents. J Fam Psychol, 25(4), 581-590. https://doi.org/10.1037/a0024148

Schill, A. (2013). http://www.family-lab.se/. Retrieved 2013 12 21, 2013, from http://www.family-lab.se/

Schultz, T. R., Stichter, J. P., Herzog, M. J., McGhee, S. D., \& Lierheimer, K. (2012). Social competence 
intervention for parents (SCI-P): Comparing outcomes for a parent education program targeting adolescents with ASD. Autism research and treatment, 2012. https://doi.org/10.1155/2012/681465

Schwarz, S. W. (2009). Adolescent mental health in the United States. National Center for Children in Poverty. New York. http://hdl.handle.net/10022/AC:P:8884

Swedish National Institute of Public Health. (2014). Health Behaviour in School-aged Children (HBSC), results from Sweden of the 2013/14 WHO study. Stockholm.: Swedish National Institute of Public Health.

Whittle, S., Vijayakumar, N., Dennison, M., Schwartz, O., Simmons, J. G., Sheeber, L., \& Allen, N. B. (2016). Observed Measures of Negative Parenting Predict Brain Development during Adolescence. PloS one, 11(1), e0147774. https://doi.org/10.1371/journal.pone.0147774

WHO. (2016). Finding the gaps in meeting adolescent health needs in Nepal. Retrieved 1 Dec, 2016, from http://www.who.int/features/2016/adolescent-health-nepal/en/

von Knorring, A-L., Cloninger, C. R., Bohman, M., \& Sigvardsson, S. (1983). An adoption study of depressive disorders and substance abuse. Archives of General Psychiatry, 40(9), 943-950. https://doi.org/10.1001/archpsyc.1983.01790080025003

Zetterqvist, M., Lundh, L-G., Dahlström, Ö., \& Svedin, C. G. (2013). Prevalence and function of non-suicidal self-injury (NSSI) in a community sample of adolescents, using suggested DSM-5 criteria for a potential NSSI disorder. Journal of abnormal child psychology, 41(5), $759-773$. https://doi.org/10.1007/s10802-013-9712-5

\section{Copyrights}

Copyright for this article is retained by the author(s), with first publication rights granted to the journal.

This is an open-access article distributed under the terms and conditions of the Creative Commons Attribution license (http://creativecommons.org/licenses/by/4.0/). 